\title{
Response time patterns in a stated choice experiment
}

\author{
Börjesson, Maria; Fosgerau, Mogens
}

Published in:

Journal of Choice Modelling

Link to article, DOI:

10.1016/j.jocm.2015.02.001

Publication date:

2015

Document Version

Peer reviewed version

Link back to DTU Orbit

Citation (APA):

Börjesson, M., \& Fosgerau, M. (2015). Response time patterns in a stated choice experiment. Journal of Choice Modelling, 14, 48-58. https://doi.org/10.1016/j.jocm.2015.02.001

\section{General rights}

Copyright and moral rights for the publications made accessible in the public portal are retained by the authors and/or other copyright owners and it is a condition of accessing publications that users recognise and abide by the legal requirements associated with these rights.

- Users may download and print one copy of any publication from the public portal for the purpose of private study or research.

- You may not further distribute the material or use it for any profit-making activity or commercial gain

- You may freely distribute the URL identifying the publication in the public portal

If you believe that this document breaches copyright please contact us providing details, and we will remove access to the work immediately and investigate your claim 


\title{
Response Time Patterns in a Stated Choice Experiment
}

\author{
Maria Börjesson $^{1}$ \\ Mogens Fosgerau ${ }^{1,2}$ \\ ${ }^{1}$ Centre for Transport Studies \\ Royal Institute of Technology \\ 10044 Stockholm, Sweden \\ ${ }^{2}$ Technical University of Denmark
}

\begin{abstract}
This paper studies how response times vary between unlabeled binary choice occasions in a stated choice (SC) experiment, with alternatives differing with respect to in-vehicle travel time and travel cost. The pattern of response times is interpreted as an indicator of the cognitive processes employed by the respondents when making their choices. We find clear signs of referencedependence in response times in the form of a strong gain-loss asymmetry. Moreover, different patterns of response times for travel time and travel cost indicate that these attributes are processed in different ways by respondents. This may be of particular relevance for choice experiments in the transportation field, where the travel time attribute is central.
\end{abstract}

Keywords: Response Times; Stated Choice; Data collection; Value of time

${ }^{1}$ maria.borjesson@abe.kth.se

2 mf@transport.dtu.dk 


\section{INTRODUCTION}

This paper studies how response times vary between choice occasions in a stated choice (SC) experiment. A general property of brain processes is the focus on gains and losses in relation to a reference point (Palmer, 1999; Kahneman, 2003). Prospect theory (Tversky and Kahneman, 1991) holds that losses matter more than equal sized gains - this effect is called loss aversion. Moreover, Tom et al. (2007) show that different parts of the brain are involved in evaluating monetary gains and losses. Many parts of the brain show increasing activity as potential gains increase, whereas losses result in decreased activity in the same parts of the brain. Thus gains and losses are processed differently. The difference in the processing of gains and losses may lead to differences in processing times.

The stated choice experiment that we use comprised unlabelled binary choice situations differing with respect to in-vehicle travel time and travel cost. The choice situations were framed as variations around a reference time and cost of a recently made trip, resulting in choices between gains and losses in travel time and travel cost. Respondents were instructed to have in mind the specific situation of their reference trip and they were specifically instructed to take into account the time constraints they faced. Each respondent made eight choices and response times were registered for each choice except the first and the last.

This paper explores how the response time of each choice is influenced by a number of variables related to the design of the experiment using both non-parametric and parametric modelling techniques. We find that response times differ systematically depending on the size of the difference of travel times and costs from the reference trip and that gains and losses affect response times asymmetrically.

More specifically, we find that response times increase significantly with reference time and decrease with reference cost. Response times also increase with the differences in travel time between the two alternatives, primarily for the choice types involving time losses relative to the reference. The response time, on the other hand, decreases with larger difference in travel cost between the two alternatives. The effect is largest for choice types implying money losses.

Reference-dependence and gain-loss asymmetry has previously been found in models estimated on similar data where the dependent variable is the actual choice and not the response time as here (Bates and Whelan, 2001; Hultkrantz and Mortazavi, 2001; Cantillo et al., 2006; De Borger and Fosgerau, 2008; Daly et al., 2011; Hjorth and Fosgerau, 2011; Börjesson and Eliasson, 2014). The finding that also response times exhibit reference-dependence and gain-loss asymmetry gives further support for application of models allowing for reference-dependence against models that do not. Existence of reference-dependence in stated choice experiments implies that such experiments do not (directly) reveal long-term stable preferences. Standard welfare economic analysis is, however, based on reference-free long-term stable preferences.

This paper is the first to look for patterns related to reference-dependence in stated choice survey response times. Previous studies of response times in stated choice surveys were all based on the hypothesis that response times correlate with quality of responses and response error. Malhotra (2008) studies response times of questions in self-administered online stated choice experiments using the response time as a measure of the cognitive effort invested by respondents (as suggested by Klein and Yadav (1989)). Malhotra argues that studies of respondents' survey engagement are increasingly 
relevant given the fast increase in the use of online surveys and in particular when using web panels rewarded for survey tasks. Also Rose and Black (2006) start out from the hypothesis that response times reflect the cognitive effort of the respondents, which in turn then would affect the quality of their choices in SC experiments. They explore the link between response times and data quality by including interactions between response times and the mean and variance of the random parameter estimates, finding it significant in many cases. This paper does not analyse the discrete choice responses and hence the issue of data quality is not so relevant in the present context.

Because response times has been interpreted as a measure of data quality, a related branch of literature exploring response times has focused on response scale heterogeneity, and how to separate this from heterogeneity in random coefficients (Louviere et al., 2002; Louviere and Eagle, 2006; Louviere et al., 1999; Swait and Bernardino, 2000). Hess and Rose (2012) show, however, that scale heterogeneity cannot be identified separately from random heterogeneity in preferences. Hess and Stathopoulos (2013) reject the idea of linking indicators of response effort (typically response time and qualitative statements) directly to the response scale since this may lead to endogeneity bias. Instead they estimate a model structure where the survey engagement is allowed to influence the response scale, and where the survey engagement is a latent variable influenced by both reported survey understanding and the response time. They also try to identify scale heterogeneity from heterogeneity in individual coefficients.

The paper is organized as follows. Section 2 describes the data. Section 3 describes a non-parametric regression technique used to generate plots and a parametric fixed effects model with response time as dependent variable. Section 4 presents the estimation results: we first apply non-parametric regression to residuals from a fixed effect regression in order to explore the properties of our data and then proceed to estimate fixed effect regression models. Section 5 concludes.

\section{DATA}

The data used in this study originate from a stated choice survey conducted in Sweden in 2008. The survey comprised car, long and short distance train and bus modes. For the car mode, a sample of respondents was drawn from the population register. They were contacted by letter and asked to participate using the internet questionnaire. Non-respondents were contacted by telephone and asked to participate either via the internet or in a call-back telephone interview. The latter option was available to avoid a potential selection bias and low response rate due to sole use of the internet. In the questionnaire, respondents were asked to list all car trips on a pre-specified day, from which one car trip was randomly selected. Selection probabilities were higher for long distance trips. The choice experiment was then framed around this selected trip.

For the public transport modes, respondents were recruited on board by collecting passengers' addresses and telephone numbers. They were asked to respond to the questionnaire via the internet or in a call-back telephone interview, and non-respondents were reminded by telephone. The public transport travellers' the choice experiment was framed around the observed trip.

In the telephone interviews, the time and costs levels of each stated choice question were read out by the interviewer. To help the respondents visualizing the alternatives, they were supplied with paper sheets on which stated choice questions with empty spaces for time and cost levels were pre-printed. The respondents were instructed to fill in the time and cost levels read out over the telephone before stating their choice. In the internet survey the alternatives were directly visible on the screen. 
For car, a total response rate of 59 percent $^{3}$ was reached and for long distance and regional public transport the response frequency varied between 70-75 percent. The number of respondents by travel mode and survey method is shown in Table 1.

Table 1: Number of respondents by travel mode and survey method.

The stated choice experiment comprised unlabelled binary choice situations differing with respect to in-vehicle travel time and travel cost. One alternative is faster but more expensive than the other, such that a price of travel time is implicit in each choice and such that there are no dominant choices in the design. In the analysis the alternatives are arranged so that the travel time and cost of the slowest and cheapest alternative are denoted $t_{1}$ and $c_{1}$, and the travel time and cost of the fastest and most expensive alternative is denoted $t_{2}$ and $c_{2}$. The implicit price of time in each bid is denoted $V=$ $-\left(c_{1}-c_{2}\right) /\left(t_{1}-t_{2}\right)$ which is always positive. Figure 1 exemplifies the choice situations faced by the interviewees. The choice indicator $l$ is defined with the convention that $l=1$ if the slow and cheap alternative is chosen (and the bid is rejected), $l=2$ if the fast and expensive alternative is chosen (the bid is accepted) and $l=3$ if the response "both alternative are equally good" is chosen.

The binary choice situations are constructed as variations around the respondent's selected (for car) or observed (for public transport) trip, which is treated as a reference point. The reference travel time is denoted $t_{r}$ (this coincides with $t_{1}$ or $t_{2}$ ) and the reference cost is denoted $c_{r}$ (this coincides with $c_{1}$ or $c_{2}$ ). From the reference trip, four types of choice situations are constructed, corresponding to the quadrants in Figure 2. The willingness to pay (WTP) choices are choices between the reference trip and an alternative that is faster and more expensive. The line in the WTP quadrant represents an example of such a choice situation: the endpoints of the line indicate the choice alternatives, one of which is the reference point. The slope of the line is the price of time implicit in this choice situation; the slope is negative, reflecting that one alternative is faster but more expensive than the other. All WTP choice situations look like this. The willingness-to-accept (WTA) choice situation is the exact opposite, comparing the reference trip to an alternative that is slower but less expensive. The line in the WTA quadrant in Figure 2 indicates an example of a WTA type choice situation. The equivalent gain $(E G)$ choice situation compares an alternative with reference time and a cost lower than the reference to an alternative with reference cost and a travel time shorter than the reference. The equivalent loss $(E L)$ choice situation is the exact opposite, including an alternative with the reference cost and a time longer than the reference and an alternative with reference time that is more expensive than the reference. In the same way as before, the lines in the EG and EL quadrants in Figure 2 indicate examples of these types of choice situations. All choice types then involve an alternative that is faster and more expensive than the other and there are hence no dominated alternatives in the design.

Figure 1: Survey question.

Figure 2: Four types of binary choice situations relative to a reference point in the origin.

\footnotetext{
${ }^{3}$ This frequency refers both to respondents in the target population and those not in the target population. The target population consisted of those who made a car trip as driver the survey day. It is likely that those in the target population had a higher response frequency than 59 percent.
} 
The experiment was designed such that each respondent made eight choices; two choices of each type. The design generated eight time differences in the $10-30$ percent range of the reference travel time, divided into four strata ${ }^{4}$. Two travel time differences were randomly assigned to each of the four quadrants. Eight values of time bids were drawn from 6 value of time strata ${ }^{5}$ in the range $0.5-50$ EUR/h and assigned randomly to each of the eight time differences. The absolute cost difference was then found for each choice situation by multiplying the absolute time difference by the trade-off value of time. The order of the four different choice types was scrambled such that the sequence of choice type is completely random.

The response times were recorded for all respondents (both telephone and internet interviewees) except for the first of the eight choice occasions. ${ }^{6}$ The telephone response time includes the time it took for the interviewer to read out the times and costs within each choice, for the respondent to fill these into the supplied paper sheets (assuming that he/she used these as instructed), the time it took for the respondent to state his/her response, and the time it took for the interviewer to feed the response into the computer.

The response time distribution for internet and telephone responses is shown in Table 2. In general the response time is higher for the telephone sample because the reading out of the times and costs in each choice situation as well as the responses over the telephone took some additional time. Since the response times in the telephone sample include additional time for reading out attribute levels, they are not directly comparable to the response times of the internet sample. The telephone sample is excluded from further analyses in this paper. ${ }^{7}$

\section{Table 2: Response time distributions.}

The total number of observations (choices made by the interviewees) is 20,664. In 259 cases the response time is 60 seconds or above and in 3 cases the recorded response time is 0 seconds. If the response time is above 60 seconds we assume that the respondent has been interrupted for a longer time and if the response is instantaneous we assume that she did not attempt to respond to the choice seriously. These observations (approximately 1 percent of the total sample) are therefore treated as outliers and discarded, leaving 20,402 observations in the sample. As a test, we have tried leaving these outliers in the dataset and this lead to no significant change in results or conclusions.

\footnotetext{
${ }^{4}$ Strata of time differences (percentages of observed travel time): 2 draws in [10\%-15\%], 2 draws in [15\%-20\%], 2 draws in [20\%-25\%], and 2 draws in [25\%-30\%].

${ }^{5}$ Strata of bids (EUR/h): 1 draw in [0.5-1.5], 1 draw in [1.5-4], 2 draws in [4-10], 2 draws in [10-20], 1 draw in [20-40], 1 draw in [40-50].

${ }^{6}$ Time stamps were recorded for every response in the survey. The time up to the first stated choice response includes time to read the instructions for the stated choice exercise and so this response is omitted. The eighth response time is not usable due to a programming error.

${ }^{7}$ Including the telephone sample would not lead to any significant changes in results or conclusions.
} 


\section{MODELLING}

This section describes the parametric and non-parametric analysis undertaken to explore the systematic relationships between the response time and the design variables. Differences in response times arising from interruptions and other noise in the data are captured by a random error. The parametric models, including the model described in Section 3.2, always require assumptions regarding the structure of the data. Non-parametric techniques such as the one described in Section 3.1, however, enable us to explore the properties of data while imposing only minimal assumptions.

\subsection{Non-parametric regression for graphical analysis}

We use local constant regression in two dimensions to explore how the response time depends on cost and time differences $\left(\log \left(c_{2}\right)-\log \left(c_{1}\right)\right)$ and $\left(\log \left(t_{1}\right)-\log \left(t_{2}\right)\right)$. The logarithmic form is chosen as it leads to better model fit in the parametric models (see below) and the non-parametric model produces plots that are easier to interpret than when using the variables in levels. Separate nonparametric regression plots are made for each of the gain-loss quadrants shown in Figure 2.

Local constant regression estimates a non-parametric function as a weighted average of a dependent variable $z$ in the neighbourhood of the independent variables $x=\left(x_{1}, x_{2}\right)$ :

$$
E(z \mid x) \approx \sum_{n} k_{n} z_{n}
$$

where $k_{n}$ is a local weight around the point $x$

$$
k_{n}=\frac{K\left(\frac{x_{n}-x}{h}\right)}{\sum_{m} K\left(\frac{x_{m}-x}{h}\right)}
$$

and $n, m$ index observations in the data. We use a standard normal density product kernel, $K\left(\frac{x_{n}-x}{h}\right)=$ $\varphi\left(\frac{x_{n 1}-x_{1}}{h}\right) \varphi\left(\frac{x_{n 2}-x_{-} 2}{h}\right)$. The bandwidth $h$ determines the size of the neighbourhood over which to average. The bandwidth is chosen by eye-balling such that the resulting plots indicate an appropriate number of features (Pagan and Ullah, 1999). For the purpose of producing plots, the estimate $E(z \mid x)$ is computed for each $x=\left(\log \left(c_{2}\right)-\log \left(c_{1}\right), \log \left(t_{1}\right)-\log \left(t_{2}\right)\right)$ taking values on a rectangular grid. The variable $z$ is the residual of an initial regression of response times on a number of other covariates as using fixed effect linear regression, which is introduced next.

\subsection{Fixed effect linear regression}

We apply a linear regression model with fixed effects to estimate the relationship between the dependent variable log of response time, denoted $y_{i t}$, observed for individual $i$ in choice situation $t$ (within the sequence of eight choice situations), and a number of explanatory variables, $X_{i t k}$. The model takes the form

$y_{i t}=\beta_{1} x_{i t 1}+\cdots+\beta_{p} x_{i t k}+\alpha_{i}+\epsilon_{n t}, \quad i=1, \ldots, N ; t=1, \ldots, T$, 
where $\alpha_{i}$ is an individual specific fixed effect and $\epsilon_{n t}$ is a normally distributed random error. The fixed effects model allows for arbitrary dependence between (time independent) unobserved individual specific variables and the controls $X_{i t}$, which, if present, would violate the assumption of the OLS model and bias the results. ${ }^{8}$

We include a number of design variables among the controls in $X$ : dummy variables for three of the four choice types (WTP, EL, EG and WTA) and the log of time and cost differences in each choice situation $\left(\log \left(c_{2}\right)-\log \left(c_{1}\right)\right)$ and $\left(\log \left(t_{1}\right)-\log \left(t_{2}\right)\right)$ for different choice types (implying gains or losses). Note that these differences are always positive because the alternatives are arranged so that the travel time and cost of the slowest and cheapest alternative are denoted $t_{1}$ and $c_{1}$. We also include the reference time $\left(t_{r}\right)$ and reference cost $\left(c_{r}\right)$ for different choice types, and dummy variables for each but one of the seven choice occasions for which the response time is registered (for the first of the eight choices it is not). The effect of the choice outcome (accept, reject and "both alternatives are equally good") is also explored.

It is possible that switching from one choice type to another induces an extra effort that increases the response time. However, since the sequence of choice types is randomized, this would not lead to a systematic effect on response times by choice type. There could still be an effect that we could detect, so we have added a dummy variable taking the value 1 if the present choice type equals the choice type of the previous choice and 0 otherwise. This variable, however, was not significant and is therefore not included in the final models presented in the paper.

\section{RESULTS}

\subsection{Graphical analysis}

Figure 3 visualizes the results from the local constant regression carried out to examine how the response time depends on time and cost differences in each quadrant. The plots are produced by first regressing log of response time $(y)$ on three quadrant dummies (EL, EG and WTP), log of reference time $\left(\log \left(t_{r}\right)\right)$ and $\log$ of reference cost $\left(\log \left(c_{r}\right)\right)$, applying fixed effects linear regression specified by (2). The variables to be explored, $\log \left(c_{2}\right)-\log \left(c_{1}\right)$ and $\log \left(t_{1}\right)-\log \left(t_{2}\right)$ are not included in this initial regression. This removes the mean log response time for each respondent, any systematic differences between quadrants and any systematic linear effect of the logs of reference time and cost. The residuals of the initial regression are then regressed against $\log \left(c_{2}\right)-\log \left(c_{1}\right)$ and $\log \left(t_{1}\right)-$ $\log \left(t_{2}\right)$, using local constant regression in two dimensions specified by (1). The resulting plots show how the residual response time depends on time and cost differences without imposing any specific functional form.

The residuals sum to zero within all choice types since the parametric model includes dummies for three of four quadrants. All of the plots are, however, not centred on zero, because the time and cost difference of the observations are not spread out uniformly over the grid; small time and cost differences are overrepresented.

\footnotetext{
${ }^{8}$ If the parameter of the independent variables changes between the OLS and the fixed effects model, this is a sign that the random error is not independent from the regressors in the OLS model, which violates a necessary assumption of this model.
} 
For all choice types, the response time decreases with the cost difference $\left(\log \left(c_{2}\right)-\log \left(c_{1}\right)\right)$. The effect seems to be largest for the EG and the EL quadrants. There is a tendency that the response time increases with time differences $\left(\log \left(t_{1}\right)-\log \left(t_{2}\right)\right)$ for choices involving time losses (WTA and EL quadrants). For the other choice types this effect is not evident from the plots.

The curves on the plots are roughly linear and equidistant, which indicates that the response time depends approximately linearly on log cost and time differences. We therefore include the log cost and time differences in the fixed effect regression in the next section. ${ }^{9}$ Moreover, the dependence varies by quadrant, which is also taken into account in the fixed effects regression.

Figure 3: Local constant regression visualizing how the response time depends on time and cost differences.

\subsection{The fixed effects regression model}

In the next step of the analysis, fixed effects regression models are estimated to simultaneously explore the effect of a number of design variables on the response times. Our key interest lies in the impact of the time and cost for gains and losses for each choice type, explored by the plots in the previous subsection. The variables for references time $\left(t_{r}\right)$ and reference cost $\left(c_{r}\right)$ are interacted with dummies for choice types. The interaction is, however, left out for one of the choice types, WTA, since the variables would otherwise be perfectly correlated with the individual specific constant. The parameters $E L \cdot \log \left(t_{0}\right), E G \cdot \log \left(t_{0}\right)$ and $W T P \cdot \log \left(t_{0}\right)$ should hence be interpreted relative to the fixed effect $\alpha_{i}$ for each individual.

Table 3 shows two fixed effects regression models specified by (2). The two models are identical except that the model to the right excludes the two dummy variables for choice outcome (one dummy for accepting the bid and one dummy for stating that "both choices are equally good"). Including these dummy variables could be problematic since they are endogenous. However, it turns out they have only slight influence on the other parameter estimates.

Both models show a systematic learning effect: relative to the first question in the sequence with recorded response time, the response time reduces systematically through the choice occasions. None of the choice type dummies are significant.

The interactions between reference time $\left(t_{r}\right)$ the dummies for the WTP, EL and EG quadrants are all positive and significant. This means that response time for these choice types, relative to WTA type choices, increases with reference time. The interactions between the reference cost $\left(c_{r}\right)$ and the dummies for the WTP and EL quadrants are negative and significant, implying that the response time of WTP and EL choices, relative to WTA choices, reduces with reference cost. The reference time and reference cost are likely to be correlated, which could induce a spurious relationship in the estimation. However, the correlation is 0.17 which is not high and the t-statistics for the corresponding parameters are reasonably high, so we do not find it likely that the estimated relationships are spurious.

The parameters for travel time and travel cost differences between the two alternatives in each type of choice are similar in both models. In consistency with the plots in Figure 3, the response time

\footnotetext{
${ }^{9}$ There is some curvature in evidence in Figure 3. We have tested specifications with quadratic terms in the fixed effect regressions and found that some of them would be significant. For ease of interpretation we stick with the linear specification in the fixed effect regressions, having confirmed that no conclusions are affected by this.
} 
decreases with larger difference in travel cost for all choice types. According to Table 3, the effect of the travel cost difference on the response time is largest for choice types involving money losses (EL and WTP). This is not entirely consistent with the slopes on cost differences in Figure 3, where the effect seems to be largest for EG and the EL quadrants. This inconsistency is due to the uneven distribution of observations in the grid of the plots (the concentration of observations is higher for small time and cost differences). The effect of travel time difference on the response time for different choice types is consistent with the plots: the effect is larges for the choice types involving time losses (WTA and EL quadrants).

The choice outcome correlates with the response time: the choice occasions where the alternative "both choices are equally good" is chosen are significantly slower than other responses. This suggests that the "equally good"-alternative is not primarily used as an option for respondents not wanting to play the game, and that response times tend to increase when the implicit trade-off price of time is close to the respondents value of time. Response times for "reject" responses are not significantly different from response times for "accept" responses. ${ }^{10,11}$

We have also estimated a plain OLS model on the controls $Y$, including all variables included in the fixed effects models shown in Table 3 and two additional variables that could not be included in the fixed effects model because they would then be perfectly correlated with the individual specific constant: the interaction terms WTA $\cdot \log \left(t_{0}\right)$ and WTA $\cdot \log \left(c_{0}\right)$. Comparison of an OLS model and a fixed effects model with otherwise identical specifications indicates, however, that the OLS model suffers from endogeneity problems: some of the estimates (the time and cost differences) differ notably (20-30\%). The parameter estimates of the model are shown in the appendix: The effects are in general consistent with the corresponding fixed effect model 1 in Table 3. The effect of $\log \left(t_{0}\right)$ is positive and significant for choice occasions involving time losses (EL and WTP) but not significantly different from zero for the other quadrants. The effect of $\log \left(c_{0}\right)$ is positive and significant for choice occasions involving time gains (EG and WTA) but not significantly different from zero for the other quadrants.

Finally we test whether the variance of the residual in the fixed effects and the OLS models depends on the controls. This checks the degree to which the observed patterns in response times are heterogeneous across respondents. The variance of the residual equals the expected value of the squared residuals of the model. ${ }^{12}$ The squared residuals are therefore regressed on the controls. This specification reveals how the variance of the unexplained response time depends on the controls. The estimates are shown in the appendix. Some of the coefficients for choice sequence are significant, implying that the learning effect varies significantly between respondents. The effect of the choice outcome "equally good" is also significant, as is the effect of travel time difference for three out of

\footnotetext{
${ }^{10}$ Moreover, the response time does not significantly correlate with the estimated value of time. This value of time model is not shown in this paper.

${ }^{11}$ Any first-order effect of socio-economic background variables on response times is captured by the fixed effect. Prompted by a reviewer, we have tried extending the fixed effect regression with interactions of gender, age and income with the cost and the time differences. The parameters included in the model shown changed only slightly and there is no impact on the conclusions that we draw. Apparently most of the effect of the socioeconomic attributes is already captured by the fixed effect.

${ }^{12}$ We assume $y=f(x)+\epsilon$, where $E(\epsilon)=0$ and the residual is independent of $x$. If the estimate of $\mathrm{y}$ is $\hat{y}$, the residuals of our models equal $\hat{\epsilon}=y-\hat{y}$. The expected value of the squared residuals is then $E\left(\hat{\epsilon}^{2} \mid x\right)=$ $E\left(y^{2}+\hat{y}^{2}-2 y \hat{y} \mid x\right)=E\left(\epsilon^{2} \mid x\right)=V(\epsilon \mid x)$.
} 
four quadrants. Hence these variables also influence the variability of response times. Otherwise there is not much evidence of heterogeneous effects of the controls on response times.

Table 3: Estimation results of the fixed effects models. Times are given in seconds and costs in SEK.

\section{DISCUSSION}

We find that response time decreases with the cost difference, most when a more expensive alternative is compared to the reference, and that response time increases with the time difference, most when a slower alternative is compared to the reference. Thus we have clear reference-dependence in response times; the dependence is asymmetric for gains and losses and goes in opposite directions for cost and time differences.

First, it is clear that the cost and time attributes are processed differently by our respondents. Previous literature has also found that different attributes are processed differently (Higgins, 1996; Kahneman, 2003).

Tom et al. (2007) show that evaluation of gains or losses activates different parts of the brain. We also find clear differences in response times between gains and losses. They moreover find that response times are longer for trade-offs that are more evenly balanced. We find the same pattern in that respondents who indicate that alternatives are equally good take longer time to reach that decision.

A longer response time indicates that more processing is going on. Our results thus indicate that larger time differences and especially time losses lead to more processing, while the opposite is the case for cost differences.

Money and time are very different things. Time cannot be stored and hence evaluating a change in travel time requires respondents to consider how to use a travel time saving or how to reschedule to accommodate a travel time increase. Time constraints vary between trips, which makes the travel time attribute context dependent. ${ }^{13}$ Rescheduling activities on a specific day is a complicated problem that requires respondents to consider their concrete activities on that day. As explained in Section 2, the present experiment, as most SC experiments, was framed around an observed recent reference trip. This setting is emphasizing both the short-term choice context and the reference trip. In contrast to time, money can be stored and people are generally involved in numerous transactions involving money.

We find clear differences in the response time pattern for time and cost gains and losses. So we may interpret our findings as indicating that the time differences involved in choice situations induce respondents to reconsider their activity schedules. Large re-scheduling effort for large travel time losses is also consistent with the finding of more loss aversion in the time than in the cost dimension (e.g., De Borger and Fosgerau (2008)).

If the respondents' preferences for travel time, and in particular travel time losses, are less available in their mind than their preferences for money, it is possible that the preferences for travel time gradually

\footnotetext{
${ }^{13}$ This is indicated by the empirical finding that trip-making within people varies considerably between days (Susilo, 2005, Pas and Koppelman, 1986; Pas and Sundar, 1995; Pas, 1987; Schlich and Axhausen, 2003, Huff and Hanson, 1986).
} 
become more available in the minds of respondents over the sequence of choice occasions, i.e. that the respondents learn about their own preferences. This hypothesis was explored by interacting the time loss (and the gain) with dummies for each choice sequence number in the fixed effects model. The increase in response time with the size of travel time loss or gain does, however, not differ systematically over the sequence of choice occasions and this hypothesis can thus be rejected.

It is sometimes a worry that SC respondents might not "play the game". Then choice of the neutral alternative could indicate that respondents do not make the effort of reaching a decision. We find that the choice outcome correlates with the response time in the way that the choices where the alternative "both choices are equally good" is chosen are significantly slower than other choices. This suggests that the "equally good"-alternative is not primarily used as an option for respondents not wanting to play the game, but that they do in fact make an effort in reaching a choice.

\section{CONCLUSION}

This study has explored how response times vary between unlabelled binary choices in a stated choice experiment, with alternatives differing with respect to in-vehicle travel time and travel cost. Specifically we explore how the response times depend on the attribute levels in each alternative compared to a reference alternative. Some clear patterns emerge indicating that time and cost differences are processed differently.

We find clear signs of reference-dependence in response times and asymmetry between gains and losses. This is consistent with the findings in the previous literature of reference-dependence and loss aversion in the trade-offs between time and cost revealed by choices (De Borger and Fosgerau 2008; Hultkrantz and Mortazavi 2001; Börjesson and Eliasson 2014; Bates and Whelan 2001; Hjorth and Fosgerau 2011; Cantillo et al., 2006; Daly et al. 2011).

Our evidence is consistent with the idea that travel time and cost gains attributes are processed in different ways. It supports the idea that cost differences are easy and hence fast to process but that travel time differences require people to consider how they would reschedule in the short term, which takes more time. This observation is of particular relevance for choice experiments in the transportation field, where the objective is to measure the value of travel time in a long-term perspective.

\section{REFERENCES}

Bates, J., \& Whelan, G. (2001). Size and sign of time savings. Working Paper. Institute of Transport Studies, University of Leeds , Leeds, UK. Retrieved from http://www.its.leeds.ac.uk/

Börjesson, M., \& Eliasson, J. (2014). Experiences from the Swedish Value of Time study. Transportation Research A, 59, 144-158.

Cantillo, V., Heydecker, B., \& Ortúzar, J. de D. (2006). A discrete choice model incorporating thresholds for perception in attribute values. Transportation Research Part B: Methodological, 40(9), 807-825. doi:10.1016/j.trb.2005.11.002

Daly, A., Tsang, F., \& Rohr, C. (2011). The value of small time savings for non-business travel. Proceedings from the European Transport Conference, 2011.

De Borger, B., \& Fosgerau, M. (2008). The trade-off between money and travel time: A test of the theory of reference-dependent preferences. Journal of Urban Economics, 64(1), 101-115. doi:16/j.jue.2007.09.001 
Hess, S., \& Rose, J. M. (2012). Can scale and coefficient heterogeneity be separated in random coefficients models? Transportation, 39(6), 1225-1239. doi:10.1007/s11116-012-9394-9

Hess, S., \& Stathopoulos, A. (2013). Linking response quality to survey engagement: A combined random scale and latent variable approach. Journal of Choice Modelling, 7, 1-12. doi:10.1016/j.jocm.2013.03.005

Higgins, E. T. (1996). Knowledge activation: Accessibility, applicability, and salience. In E. T. Higgins \& A. W. Kruglanski (Eds.), Social psychology: Handbook of basic principles (pp. 133-168). New York, NY, US: Guilford Press.

Hjorth, K., \& Fosgerau, M. (2011). Loss Aversion and Individual Characteristics. Environmental and Resource Economics, 49(4), 573-596. doi:10.1007/s10640-010-9455-5

Hultkrantz, L., Mortazavi, R., \& Hulkranz, L. (2001). Anomalies in the Value of Travel-Time Changes. Journal of Transport Economics and Policy, 35(2), 285-299. doi:10.2307/20053871

Kahneman, D. (2003). Maps of Bounded Rationality: Psychology for Behavioral Economics. The American Economic Review, 93(5), 1449-1475.

Klein, N. M., \& Yadav, M. S. (1989). Context Effects on Effort and Accuracy in Choice: An Enquiry into Adaptive Decision Making. Journal of Consumer Research, 15(4), 411-421.

Louviere, J., \& Eagle, T. (2006). Confound it! That pesky little scale constant messes up our convenient assumptions. Retrieved from http://epress.lib.uts.edu.au/research/handle/10453/7515

Louviere, J. J., Meyer, R. J., Bunch, D. S., Carson, R., Dellaert, B., Hanemann, W. M., ... Irwin, J. (1999). Combining Sources of Preference Data for Modeling Complex Decision Processes. Marketing Letters, 10(3), 205-217. doi:10.1023/A:1008050215270

Louviere, J., Street, D., Carson, R., Ainslie, A., Deshazo, J. R., Cameron, T., ... Marley, T. (2002). Dissecting the Random Component of Utility. Marketing Letters, 13(3), 177-193. doi:10.1023/A:1020258402210

Malhotra, N. (2008). Completion Time and Response Order Effects in Web Surveys. Public Opinion Quarterly, 72(5), 914-934. doi:10.1093/poq/nfn050

Pagan, A., \& Ullah, A. (1999). Nonparametric Econometrics. Cambridge University Press.

Palmer, S. E. (1999). Vision science: Photons to phenomenology (Vol. xxii). Cambridge, MA, US: The MIT Press.

Rose, J. M., \& Black, I. R. (2006). Means matter, but variance matter too: Decomposing response latency influences on variance heterogeneity in stated preference experiments. Marketing Letters, 17(4), 295-310. doi:10.1007/s11002-006-8632-3

Susilo, Y. (2005). The Short-term Variability and the Long-term Changes of Individual Spatial Behavior in Urban Areas (PhD Thesis). Kyoto University.

Swait, J., \& Bernardino, A. (2000). Distinguishing taste variation from error structure in discrete choice data. Transportation Research Part B: Methodological, 34(1), 1-15. doi:10.1016/S0191-2615(99)00009-0

Tom, S. M., Fox, C. R., Trepel, C., \& Poldrack, R. A. (2007). The Neural Basis of Loss Aversion in Decision-Making Under Risk. Science, 315(5811), 515-518. doi:10.1126/science.1134239

Tversky, A., \& Kahneman, D. (1991). Loss Aversion in Riskless Choice: A Reference-Dependent Model. The Quarterly Journal of Economics, 106(4), 1039-1061. doi:10.2307/2937956

\section{APPENDIX}

Table 4: Estimation results of the fixed effects (FE) model and corresponding OLS models (the FE model 5 is identical to model 1). The log of response time is the dependent variable of models 5 and 7 and the dependent variable of models 6 and 8 is the squared residuals of models 5 and 7. Times are given in seconds and costs in SEK. 
Table 1: Number of respondents by travel mode and survey method.

\begin{tabular}{|l|r|r|r|r|r|}
\hline \hline Survey Method & Long Distance Bus & Regional Bus & Long Distance Train & Regional Train & Car \\
\hline \hline Internet & 564 & 804 & 465 & 833 & 1209 \\
\hline Telephone & 147 & 155 & 107 & 101 & 262 \\
\hline Total & 711 & 959 & 572 & 934 & 1471 \\
\hline
\end{tabular}

Table 2: Response time distributions.

\begin{tabular}{|l|r|r|r|r|r|r|}
\hline \hline & \multicolumn{1}{|c|}{ Min } & 1 Quartile & \multicolumn{1}{c|}{ Median } & Mean & 3 Quartile & \multicolumn{1}{c|}{ Max } \\
\hline \hline Internet & 1.00 & 8.0 & 11.0 & 13.0 & 15.0 & 60.0 \\
\hline Telephone & 1.00 & 11.0 & 16.0 & 17.7 & 23.0 & 60.0 \\
\hline
\end{tabular}


Table 3: Estimation results of the fixed effects models. Times are given in seconds and costs in SEK.

\begin{tabular}{|c|c|c|c|c|}
\hline Model & \multicolumn{2}{|c|}{ 1. FE } & \multicolumn{2}{|c|}{ 2. FE } \\
\hline \# respondents & & 3762 & & 3762 \\
\hline \# observations & & 20402 & & 20402 \\
\hline R-squared & & 0.1247 & & 0.0986 \\
\hline & Estimat & t- test & Estimat & t- test \\
\hline Question 3 & -0.14 & -14.71 & -0.14 & -14.69 \\
\hline Question 4 & -0.21 & -20.63 & -0.21 & -20.56 \\
\hline Question 5 & -0.33 & -35.30 & -0.33 & -35.18 \\
\hline Question 6 & -0.34 & -36.33 & -0.34 & -36.27 \\
\hline Question 7 & -0.34 & -35.84 & -0.34 & -35.76 \\
\hline EL & 0.03 & 0.87 & 0.03 & 1.02 \\
\hline EG & 0.05 & 1.54 & 0.05 & 1.50 \\
\hline WTP & 0.05 & 1.65 & 0.05 & 1.67 \\
\hline Accept & 0.00 & 0.39 & & \\
\hline Equal & 0.08 & 8.00 & & \\
\hline$E L \cdot \log \left(t_{0}\right)$ & 0.07 & 4.48 & 0.07 & 4.64 \\
\hline$E G \cdot \log \left(t_{0}\right)$ & 0.04 & 2.68 & 0.04 & 2.68 \\
\hline$W T P \cdot \log \left(t_{0}\right)$ & 0.06 & 4.07 & 0.06 & 4.17 \\
\hline$E L \cdot \log \left(c_{0}\right)$ & -0.04 & -3.58 & -0.05 & -3.74 \\
\hline$E G \cdot \log \left(c_{0}\right)$ & -0.01 & -0.87 & -0.01 & -0.89 \\
\hline$W T P \cdot \log \left(c_{0}\right)$ & -0.04 & -3.47 & -0.04 & -3.62 \\
\hline$E L \cdot\left(\log \left(t_{1}\right)-\log \left(t_{2}\right)\right)$ & 0.38 & 2.88 & 0.34 & 2.55 \\
\hline$E G \cdot\left(\log \left(t_{1}\right)-\log \left(t_{2}\right)\right)$ & 0.14 & 1.76 & 0.11 & 1.36 \\
\hline$W T P \cdot\left(\log \left(t_{1}\right)-\log \left(t_{2}\right)\right)$ & 0.07 & 0.81 & 0.03 & 0.41 \\
\hline WTA. $\left(\log \left(t_{1}\right)-\log \left(t_{2}\right)\right)$ & 0.41 & 3.36 & 0.37 & 3.05 \\
\hline$E L \cdot\left(\log \left(c_{2}\right)-\log \left(c_{1}\right)\right)$ & -0.13 & -5.22 & -0.15 & -6.25 \\
\hline$E G \cdot\left(\log \left(c_{2}\right)-\log \left(c_{1}\right)\right)$ & -0.03 & -3.36 & -0.04 & -4.16 \\
\hline$W T P \cdot\left(\log \left(c_{2}\right)-\log \left(c_{1}\right)\right)$ & -0.11 & -4.15 & -0.13 & -5.05 \\
\hline$W T A \cdot\left(\log \left(c_{2}\right)-\log \left(c_{1}\right)\right)$ & -0.01 & -1.56 & -0.02 & -2.40 \\
\hline
\end{tabular}


Table 4: Estimation results of the fixed effects (FE) model and corresponding OLS models (the FE model 5 is identical to model 1). The log of response time is the dependent variable of models 3 and 5 and the dependent variable of models 4 and 6 is the squared residuals of models 3 and 5. Times are given in seconds and costs in SEK.

\begin{tabular}{|c|c|c|c|c|c|c|c|c|}
\hline Model & \multicolumn{2}{|c|}{ 1.FE } & \multicolumn{2}{|c|}{ 4. OLS } & \multicolumn{2}{|c|}{ 5. OLS } & \multicolumn{2}{|c|}{ 6. OLS } \\
\hline Dependent variable & Respor & time & Squared & siduals & Respol & e time & Squared & siduals \\
\hline \# respondents & & 3762 & & 3762 & & 3762 & & 3762 \\
\hline \# observations & & 20402 & & 20402 & & 20402 & & 20402 \\
\hline R-squared & & 0.1247 & & 0.0058 & & 0.0713 & & 0.0070 \\
\hline & Estimat & t-test & Estimat & t- test & Estimat & t- test & Estimat & t- test \\
\hline Intercept & & & 0.08 & 6.02 & 2.70 & "90.18 & 0.19 & 6.59 \\
\hline Question 3 & -0.14 & -14.71 & -0.01 & -2.32 & -0.14 & -11.00 & 0.01 & 1.13 \\
\hline Question 4 & -0.21 & -20.63 & -0.01 & -1.79 & -0.21 & -15.42 & 0.00 & 0.30 \\
\hline Question 5 & -0.33 & -35.30 & -0.02 & -2.83 & -0.33 & -25.82 & 0.01 & 0.88 \\
\hline Question 6 & -0.34 & -36.33 & -0.01 & -1.44 & -0.34 & -26.89 & 0.01 & 1.11 \\
\hline Question 7 & -0.34 & -35.84 & 0.00 & 0.77 & -0.34 & -26.72 & 0.02 & 1.99 \\
\hline EL & 0.03 & 0.87 & 0.01 & 0.74 & -0.01 & -0.14 & 0.05 & 1.23 \\
\hline $\mathrm{EG}$ & 0.05 & 1.54 & 0.00 & 0.04 & 0.05 & 1.30 & 0.04 & 1.16 \\
\hline WTP & 0.05 & 1.65 & 0.01 & 0.41 & 0.06 & 1.61 & -0.01 & -0.17 \\
\hline Accept & 0.00 & 0.39 & 0.00 & 0.69 & -0.01 & -1.07 & 0.01 & 0.82 \\
\hline Equal & 0.08 & 8.00 & 0.04 & 8.01 & 0.00 & -0.32 & 0.11 & 10.52 \\
\hline$E L \cdot \log \left(t_{0}\right)$ & 0.07 & 4.48 & -0.01 & -1.85 & 0.07 & 5.18 & -0.03 & -2.08 \\
\hline$E G \cdot \log \left(t_{0}\right)$ & 0.04 & 2.68 & -0.01 & -1.82 & 0.02 & 1.73 & 0.00 & -0.38 \\
\hline$W T P \cdot \log \left(t_{0}\right)$ & 0.06 & 4.07 & 0.01 & 1.22 & 0.05 & 3.73 & 0.01 & 0.88 \\
\hline$W T A \cdot \log \left(t_{0}\right)$ & & & 0.00 & 0.51 & -0.01 & -0.77 & -0.01 & -0.82 \\
\hline$E L \cdot \log \left(c_{0}\right)$ & -0.04 & -3.58 & 0.01 & 1.17 & -0.01 & -0.93 & 0.03 & 2.47 \\
\hline$E G \cdot \log \left(c_{0}\right)$ & -0.01 & -0.87 & 0.01 & 1.18 & 0.04 & 4.19 & 0.01 & 1.11 \\
\hline$W T P \cdot \log \left(c_{0}\right)$ & -0.04 & -3.47 & -0.01 & -1.92 & 0.00 & -0.02 & -0.01 & -0.95 \\
\hline$W T A \cdot \log \left(c_{0}\right)$ & & & -0.01 & -2.07 & 0.05 & 4.93 & 0.01 & 0.67 \\
\hline$E L \cdot\left(\log \left(t_{1}\right)-\log \left(t_{2}\right)\right)$ & 0.38 & 2.88 & 0.05 & 0.61 & 0.55 & 3.36 & -0.03 & -0.21 \\
\hline$E G \cdot\left(\log \left(t_{1}\right)-\log \left(t_{2}\right)\right)$ & 0.14 & 1.76 & 0.10 & 1.93 & 0.03 & 0.30 & 0.03 & 0.29 \\
\hline$W T P \cdot\left(\log \left(t_{1}\right)-\log \left(t_{2}\right)\right)$ & 0.07 & 0.81 & 0.10 & 1.97 & -0.07 & -0.71 & 0.29 & 2.80 \\
\hline$W T A \cdot\left(\log \left(t_{1}\right)-\log \left(t_{2}\right)\right)$ & 0.41 & 3.36 & 0.21 & 2.86 & 0.34 & 2.23 & 0.17 & 1.16 \\
\hline$E L \cdot\left(\log \left(c_{2}\right)-\log \left(c_{1}\right)\right)$ & -0.13 & -5.22 & 0.02 & 1.58 & -0.20 & -6.38 & 0.07 & 2.46 \\
\hline$E G \cdot\left(\log \left(c_{2}\right)-\log \left(c_{1}\right)\right)$ & -0.03 & -3.36 & 0.01 & 1.22 & -0.03 & -2.95 & 0.02 & 1.50 \\
\hline$W T P \cdot\left(\log \left(c_{2}\right)-\log \left(c_{1}\right)\right)$ & -0.11 & -4.15 & -0.01 & -0.41 & -0.14 & -4.54 & -0.04 & -1.18 \\
\hline$W T A \cdot\left(\log \left(c_{2}\right)-\log \left(c_{1}\right)\right)$ & -0.01 & -1.56 & 0.00 & 0.16 & -0.03 & -2.55 & 0.02 & 1.72 \\
\hline
\end{tabular}




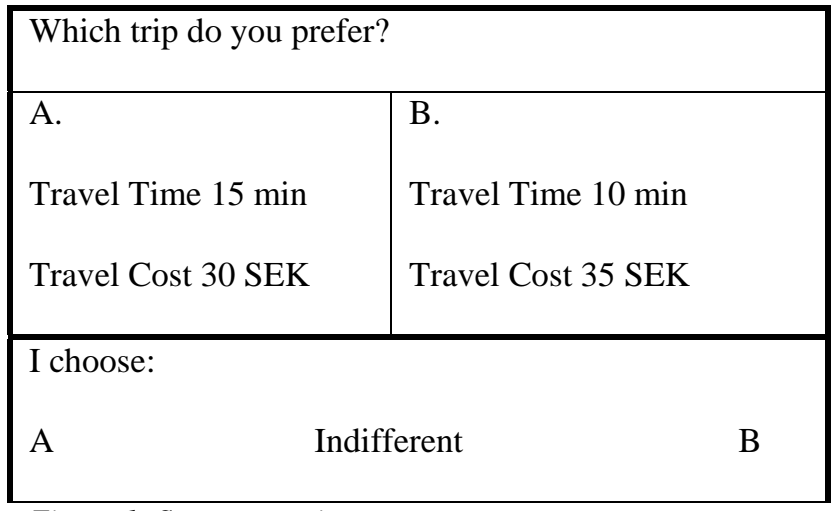

Figure 1: Survey question.

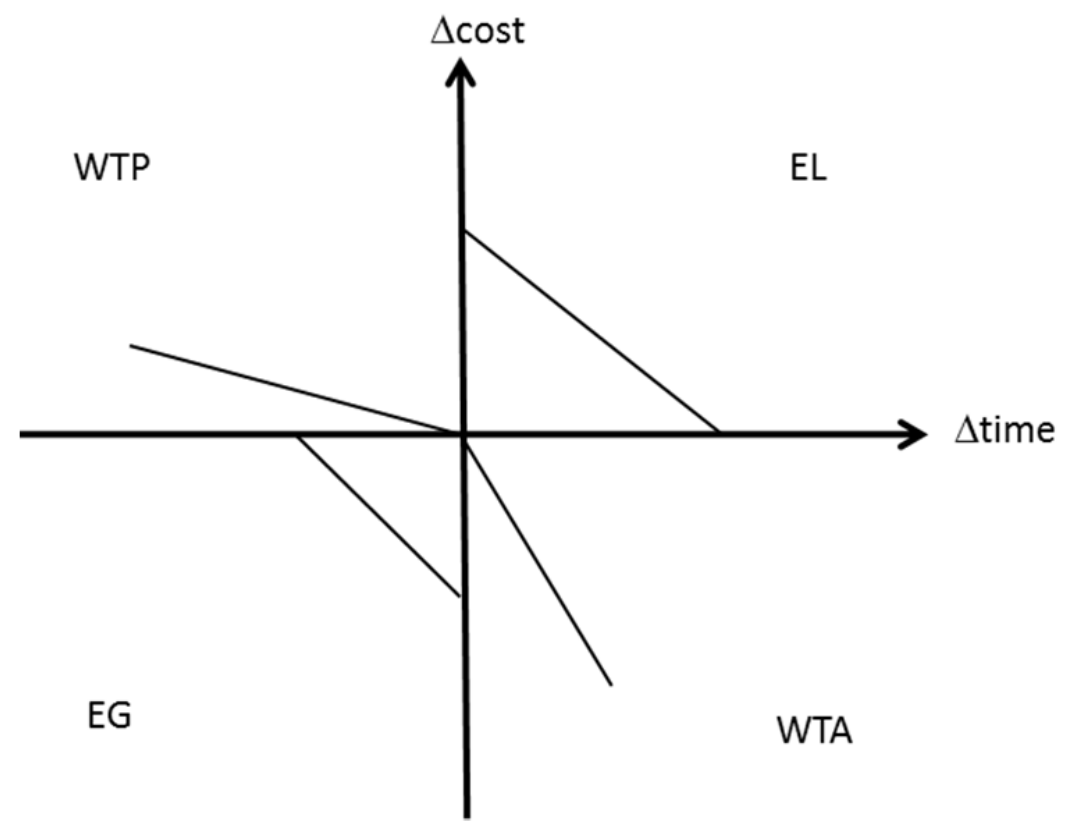

Figure 2: Four types of binary choices relative to a reference point in the origin. 

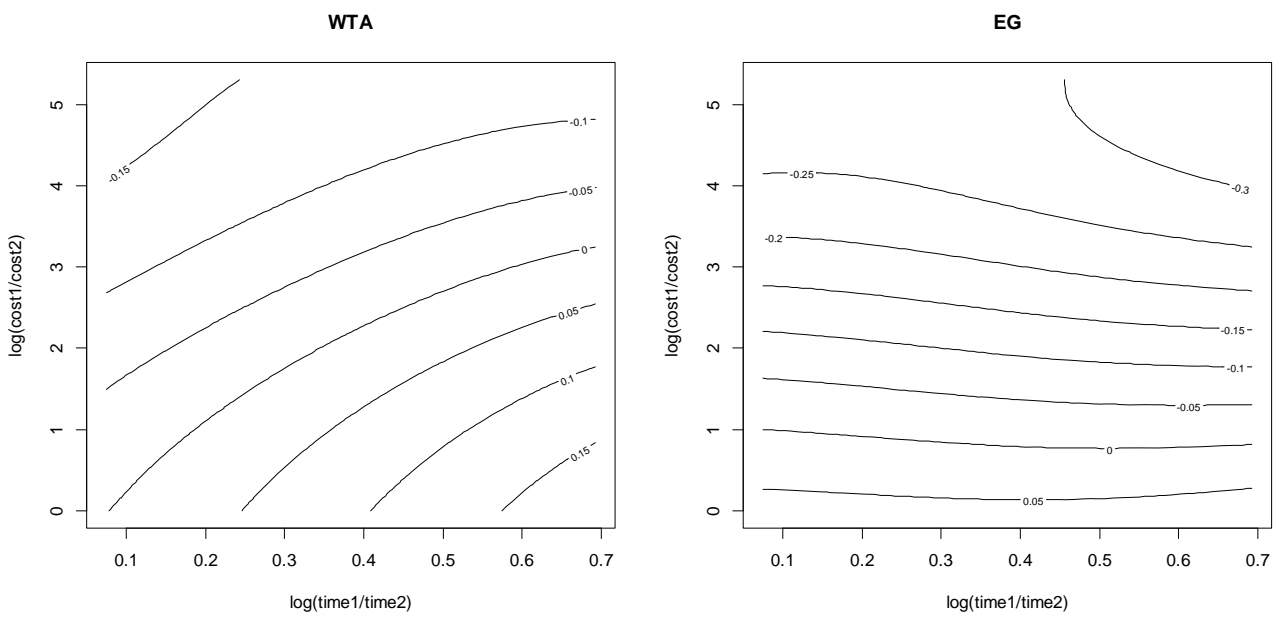

EL
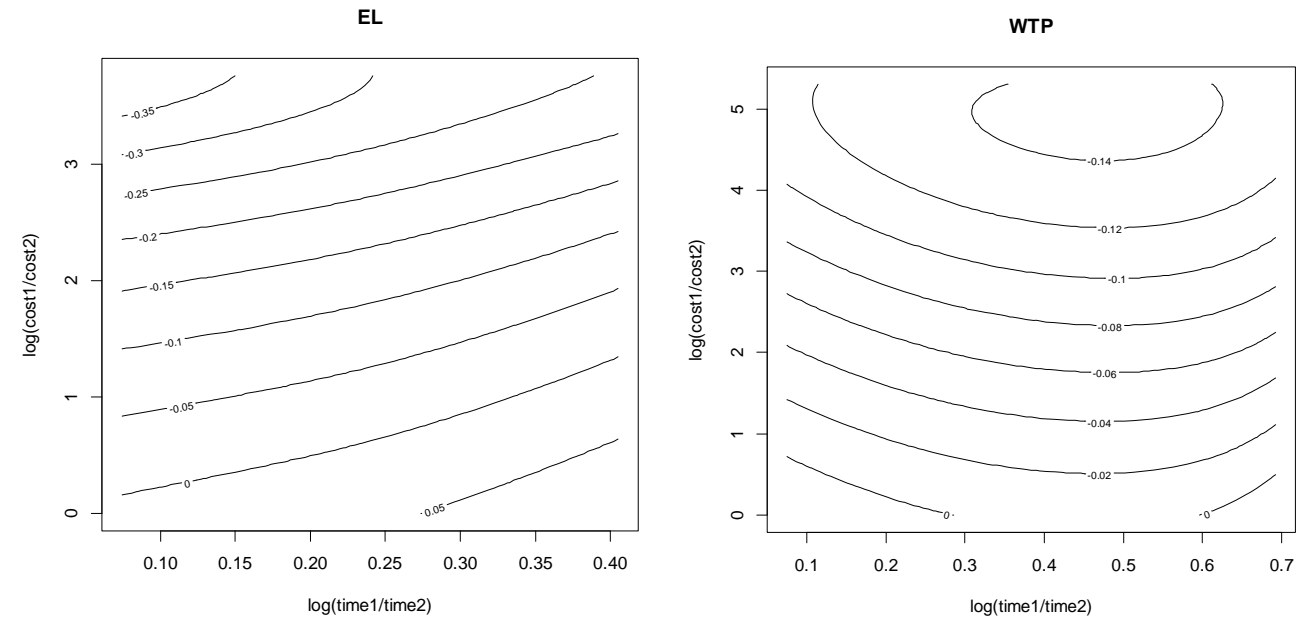

Figure 3: Local constant regression visualizing how the response time depends on time and cost differences. 
Table 1: Number of respondents by travel mode and survey method.

\begin{tabular}{|l|r|r|r|r|r|}
\hline \hline Survey Method & Long Distance Bus & Regional Bus & Long Distance Train & Regional Train & Car \\
\hline \hline Internet & 564 & 804 & 465 & 833 & 1209 \\
\hline Telephone & 147 & 155 & 107 & 101 & 262 \\
\hline Total & 711 & 959 & 572 & 934 & 1471 \\
\hline
\end{tabular}

Table 2: Response time distributions.

\begin{tabular}{|l|r|r|r|r|r|r|}
\hline \hline & \multicolumn{1}{|c|}{ Min } & 1 Quartile & \multicolumn{1}{c|}{ Median } & Mean & 3 Quartile & \multicolumn{1}{c|}{ Max } \\
\hline \hline Internet & 1.00 & 8.0 & 11.0 & 13.0 & 15.0 & 60.0 \\
\hline Telephone & 1.00 & 11.0 & 16.0 & 17.7 & 23.0 & 60.0 \\
\hline
\end{tabular}


Table 3: Estimation results of the fixed effects models. Times are given in seconds and costs in SEK.

\begin{tabular}{|c|c|c|c|c|}
\hline Model & \multicolumn{2}{|c|}{ 1. FE } & \multicolumn{2}{|c|}{ 2. FE } \\
\hline \# respondents & & 3762 & & 3762 \\
\hline \# observations & & 20402 & & 20402 \\
\hline R-squared & & 0.1247 & & 0.0986 \\
\hline & Estimat & t- test & Estimat & t- test \\
\hline Question 3 & -0.14 & -14.71 & -0.14 & -14.69 \\
\hline Question 4 & -0.21 & -20.63 & -0.21 & -20.56 \\
\hline Question 5 & -0.33 & -35.30 & -0.33 & -35.18 \\
\hline Question 6 & -0.34 & -36.33 & -0.34 & -36.27 \\
\hline Question 7 & -0.34 & -35.84 & -0.34 & -35.76 \\
\hline EL & 0.03 & 0.87 & 0.03 & 1.02 \\
\hline EG & 0.05 & 1.54 & 0.05 & 1.50 \\
\hline WTP & 0.05 & 1.65 & 0.05 & 1.67 \\
\hline Accept & 0.00 & 0.39 & & \\
\hline Equal & 0.08 & 8.00 & & \\
\hline$E L \cdot \log \left(t_{0}\right)$ & 0.07 & 4.48 & 0.07 & 4.64 \\
\hline$E G \cdot \log \left(t_{0}\right)$ & 0.04 & 2.68 & 0.04 & 2.68 \\
\hline$W T P \cdot \log \left(t_{0}\right)$ & 0.06 & 4.07 & 0.06 & 4.17 \\
\hline$E L \cdot \log \left(c_{0}\right)$ & -0.04 & -3.58 & -0.05 & -3.74 \\
\hline$E G \cdot \log \left(c_{0}\right)$ & -0.01 & -0.87 & -0.01 & -0.89 \\
\hline$W T P \cdot \log \left(c_{0}\right)$ & -0.04 & -3.47 & -0.04 & -3.62 \\
\hline$E L \cdot\left(\log \left(t_{1}\right)-\log \left(t_{2}\right)\right)$ & 0.38 & 2.88 & 0.34 & 2.55 \\
\hline$E G \cdot\left(\log \left(t_{1}\right)-\log \left(t_{2}\right)\right)$ & 0.14 & 1.76 & 0.11 & 1.36 \\
\hline$W T P \cdot\left(\log \left(t_{1}\right)-\log \left(t_{2}\right)\right)$ & 0.07 & 0.81 & 0.03 & 0.41 \\
\hline WTA. $\left(\log \left(t_{1}\right)-\log \left(t_{2}\right)\right)$ & 0.41 & 3.36 & 0.37 & 3.05 \\
\hline$E L \cdot\left(\log \left(c_{2}\right)-\log \left(c_{1}\right)\right)$ & -0.13 & -5.22 & -0.15 & -6.25 \\
\hline$E G \cdot\left(\log \left(c_{2}\right)-\log \left(c_{1}\right)\right)$ & -0.03 & -3.36 & -0.04 & -4.16 \\
\hline$W T P \cdot\left(\log \left(c_{2}\right)-\log \left(c_{1}\right)\right)$ & -0.11 & -4.15 & -0.13 & -5.05 \\
\hline$W T A \cdot\left(\log \left(c_{2}\right)-\log \left(c_{1}\right)\right)$ & -0.01 & -1.56 & -0.02 & -2.40 \\
\hline
\end{tabular}


Table 4: Estimation results of the fixed effects (FE) model and corresponding OLS models (the FE model 5 is identical to model 1). The log of response time is the dependent variable of models 3 and 5 and the dependent variable of models 4 and 6 is the squared residuals of models 3 and 5. Times are given in seconds and costs in SEK.

\begin{tabular}{|c|c|c|c|c|c|c|c|c|}
\hline Model & \multicolumn{2}{|c|}{ 1.FE } & \multicolumn{2}{|c|}{ 4. OLS } & \multicolumn{2}{|c|}{ 5. OLS } & \multicolumn{2}{|c|}{ 6. OLS } \\
\hline Dependent variable & Respor & time & Squared & siduals & Respol & e time & Squared & siduals \\
\hline \# respondents & & 3762 & & 3762 & & 3762 & & 3762 \\
\hline \# observations & & 20402 & & 20402 & & 20402 & & 20402 \\
\hline R-squared & & 0.1247 & & 0.0058 & & 0.0713 & & 0.0070 \\
\hline & Estimat & t-test & Estimat & t- test & Estimat & t- test & Estimat & t- test \\
\hline Intercept & & & 0.08 & 6.02 & 2.70 & "90.18 & 0.19 & 6.59 \\
\hline Question 3 & -0.14 & -14.71 & -0.01 & -2.32 & -0.14 & -11.00 & 0.01 & 1.13 \\
\hline Question 4 & -0.21 & -20.63 & -0.01 & -1.79 & -0.21 & -15.42 & 0.00 & 0.30 \\
\hline Question 5 & -0.33 & -35.30 & -0.02 & -2.83 & -0.33 & -25.82 & 0.01 & 0.88 \\
\hline Question 6 & -0.34 & -36.33 & -0.01 & -1.44 & -0.34 & -26.89 & 0.01 & 1.11 \\
\hline Question 7 & -0.34 & -35.84 & 0.00 & 0.77 & -0.34 & -26.72 & 0.02 & 1.99 \\
\hline EL & 0.03 & 0.87 & 0.01 & 0.74 & -0.01 & -0.14 & 0.05 & 1.23 \\
\hline $\mathrm{EG}$ & 0.05 & 1.54 & 0.00 & 0.04 & 0.05 & 1.30 & 0.04 & 1.16 \\
\hline WTP & 0.05 & 1.65 & 0.01 & 0.41 & 0.06 & 1.61 & -0.01 & -0.17 \\
\hline Accept & 0.00 & 0.39 & 0.00 & 0.69 & -0.01 & -1.07 & 0.01 & 0.82 \\
\hline Equal & 0.08 & 8.00 & 0.04 & 8.01 & 0.00 & -0.32 & 0.11 & 10.52 \\
\hline$E L \cdot \log \left(t_{0}\right)$ & 0.07 & 4.48 & -0.01 & -1.85 & 0.07 & 5.18 & -0.03 & -2.08 \\
\hline$E G \cdot \log \left(t_{0}\right)$ & 0.04 & 2.68 & -0.01 & -1.82 & 0.02 & 1.73 & 0.00 & -0.38 \\
\hline$W T P \cdot \log \left(t_{0}\right)$ & 0.06 & 4.07 & 0.01 & 1.22 & 0.05 & 3.73 & 0.01 & 0.88 \\
\hline$W T A \cdot \log \left(t_{0}\right)$ & & & 0.00 & 0.51 & -0.01 & -0.77 & -0.01 & -0.82 \\
\hline$E L \cdot \log \left(c_{0}\right)$ & -0.04 & -3.58 & 0.01 & 1.17 & -0.01 & -0.93 & 0.03 & 2.47 \\
\hline$E G \cdot \log \left(c_{0}\right)$ & -0.01 & -0.87 & 0.01 & 1.18 & 0.04 & 4.19 & 0.01 & 1.11 \\
\hline$W T P \cdot \log \left(c_{0}\right)$ & -0.04 & -3.47 & -0.01 & -1.92 & 0.00 & -0.02 & -0.01 & -0.95 \\
\hline$W T A \cdot \log \left(c_{0}\right)$ & & & -0.01 & -2.07 & 0.05 & 4.93 & 0.01 & 0.67 \\
\hline$E L \cdot\left(\log \left(t_{1}\right)-\log \left(t_{2}\right)\right)$ & 0.38 & 2.88 & 0.05 & 0.61 & 0.55 & 3.36 & -0.03 & -0.21 \\
\hline$E G \cdot\left(\log \left(t_{1}\right)-\log \left(t_{2}\right)\right)$ & 0.14 & 1.76 & 0.10 & 1.93 & 0.03 & 0.30 & 0.03 & 0.29 \\
\hline$W T P \cdot\left(\log \left(t_{1}\right)-\log \left(t_{2}\right)\right)$ & 0.07 & 0.81 & 0.10 & 1.97 & -0.07 & -0.71 & 0.29 & 2.80 \\
\hline$W T A \cdot\left(\log \left(t_{1}\right)-\log \left(t_{2}\right)\right)$ & 0.41 & 3.36 & 0.21 & 2.86 & 0.34 & 2.23 & 0.17 & 1.16 \\
\hline$E L \cdot\left(\log \left(c_{2}\right)-\log \left(c_{1}\right)\right)$ & -0.13 & -5.22 & 0.02 & 1.58 & -0.20 & -6.38 & 0.07 & 2.46 \\
\hline$E G \cdot\left(\log \left(c_{2}\right)-\log \left(c_{1}\right)\right)$ & -0.03 & -3.36 & 0.01 & 1.22 & -0.03 & -2.95 & 0.02 & 1.50 \\
\hline$W T P \cdot\left(\log \left(c_{2}\right)-\log \left(c_{1}\right)\right)$ & -0.11 & -4.15 & -0.01 & -0.41 & -0.14 & -4.54 & -0.04 & -1.18 \\
\hline$W T A \cdot\left(\log \left(c_{2}\right)-\log \left(c_{1}\right)\right)$ & -0.01 & -1.56 & 0.00 & 0.16 & -0.03 & -2.55 & 0.02 & 1.72 \\
\hline
\end{tabular}




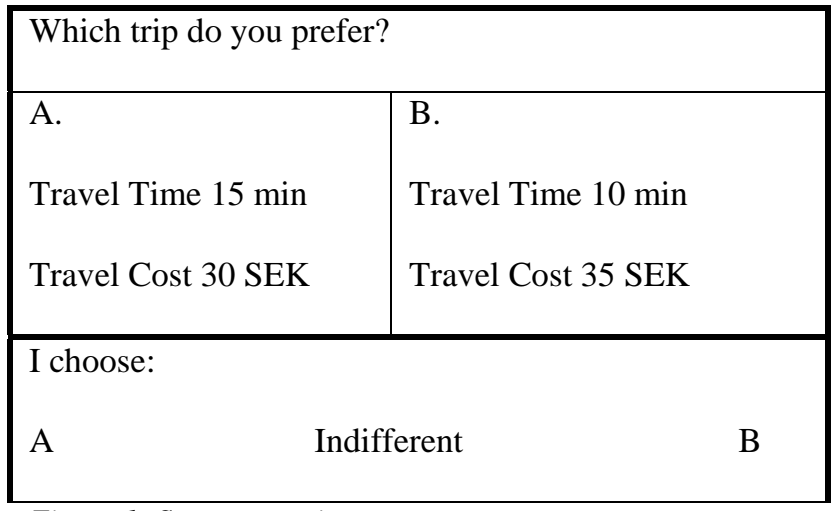

Figure 1: Survey question.

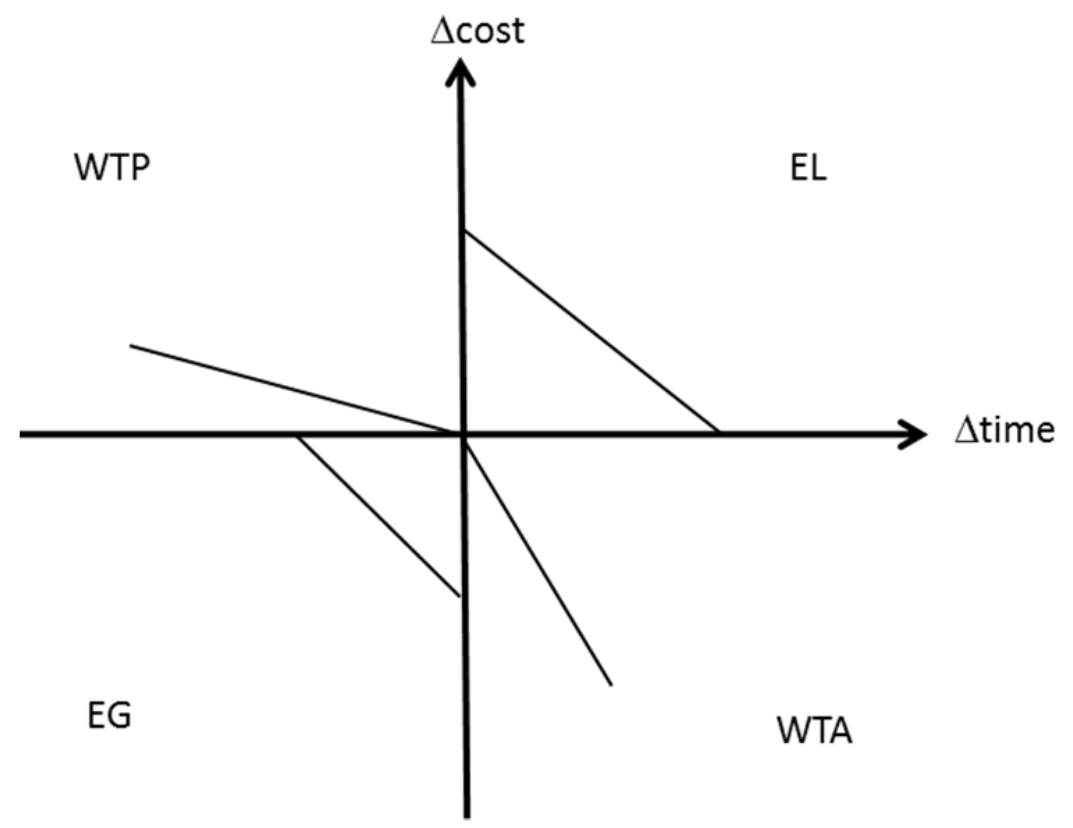

Figure 2: Four types of binary choices relative to a reference point in the origin. 

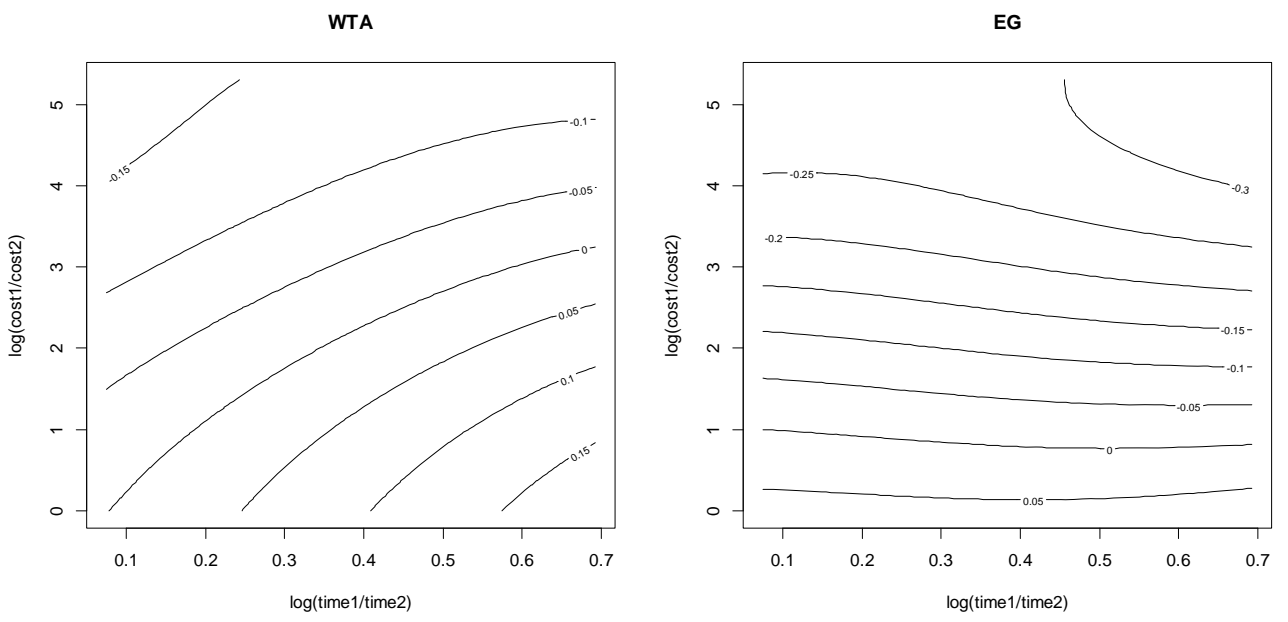

EL
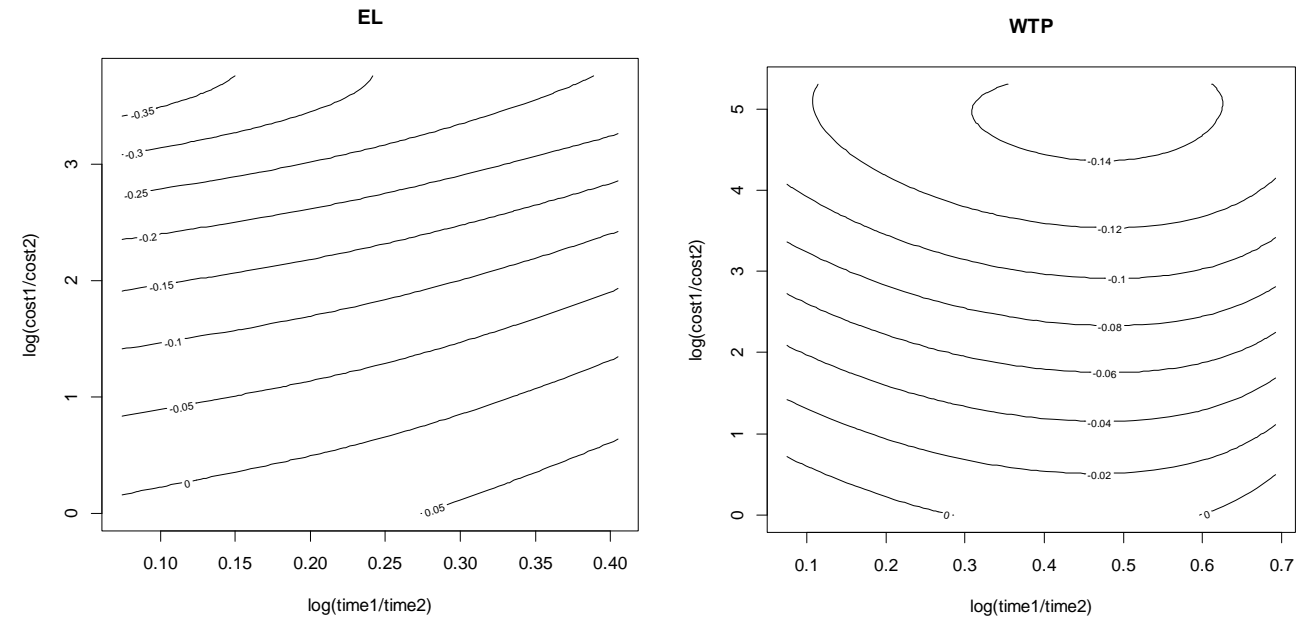

Figure 3: Local constant regression visualizing how the response time depends on time and cost differences. 